\title{
Nghiên cứu hành vi mua ngẫu hứng tại sân bay Tân Sơn Nhất
}

\author{
Hà Nam Khánh Giao \\ Khoa Vận tải Hàng không, Học viện Hàng không Việt Nam \\ Email:khanhgiaohn@yahoo.com \\ Ngày nhận bài: 17/8/2021; Ngày duyệt đăng: 05/10/2021
}

\section{Tóm tắt}

Nghiên cưu này được thực hiện nhằm phân tích tương tác giữa khuynh hướng mua

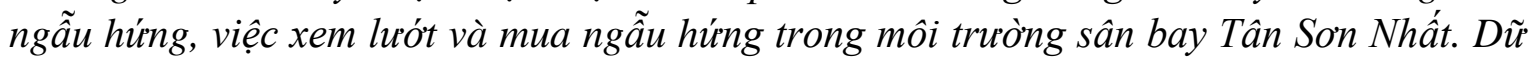
liệu thu thập tù 166 đáp viên đã mua hàng tại sân bay, bằng bảng câu hỏi cấu trúc. Phân tích hồi quy, t-test và ANOVA được sủ dụng để kiểm định mô hình đề xuất. Kết quả nghiên cưu cho thấy mặc dù khuynh hướng mua ngẫu hưng có tác động ngược chiều mua ngẫu hưng, tuy nhiên, người có khuynh hương mua ngẫu hưng cao thi có ý định xem lướt nhiều hơn. Tù đó, hành động xem lướt là rất phổ biến tại sân bay, nhung không hẳn sẽ dẫn đến mua ngẫu hứng. Điều này gợi mở nhiều ứng dụng cho nhũ̃ng nhà bán lẻ sân bay.

Tù̀ khóa: bán lẻ sân bay, hành vi mua hàng của khách hàng, khuynh huớng mua ngẫu hứng, mua ngẫu húng, xem luớt

\section{Impulse buying at Tân Sơn Nhất international airport}

\begin{abstract}
The present study has been undertaken to analyze the interrelationship between impulse buying tendency, browsing and impulse purchase in the Tan Son Nhat Airport environment. The data was collected from 166 respondents who had made a purchase at the airport using a structured questionnaire. Regression analysis, $t$-test and ANOVA was applied to test the proposed model. The results of the study indicate that although impulse buying tendency was found to have a negative association with impulse purchase, the people having high impulse buying tendency were likely to browse more. This suggests that browsing activity is common in the airport environment, but it may not necessarily lead to impulse purchase. The results provide several important implications for the airport retailers.
\end{abstract}

Keywords: airport retail, browsing, consumer buying behavior, impulse buying tendency, impulse purchase

\section{Giới thiệu}

Bán lẻ, dưới nhiều hình thức, hiện đang là một thành phần chủ lực của nền kinh tế Việt Nam, đóng góp khoảng 8\% GDP (Đỗ Nga, 2020). Bán lẻ tại sân bay là một hình thức bán lẻ khác biệt so với những kênh tiêu thụ hàng ngày khác. Tuy nhiên, bán lẻ sân bay (airport retailing) vẫn được xem là thị trường ngách và đang phát triển (Omar và Kent, 2001; Thomas, 1997; Walsh và McColl, 1994). Vì vậy, ngày nay, sân bay không còn bị coi là một nơi buồn chán, mà chuyển sang là địa điểm rất năng động. Thế hệ hành khách mới được chiều chuộng bằng 
mọi cách, và từ đó, bán lẻ sân bay cũng chuyển sang khuynh hướng mới. Trong những năm gần đây, việc thương mại hóa sân bay đã diễn ra toàn cầu, vì vậy, hành khách có xu hướng tiêu tiền nhanh chóng tại sân bay. Các sân bay đang chuyển dần từ trạng thái một vài cửa hàng đồ lưu niệm địa phương cho du khách trong những phút cuối sang một hệ thống cửa hàng đầy đủ dịch vụ lớn.

Các sân bay trở nên rất hấp dẫn cho các nhà bán lẻ vì khả năng phát triển, doanh số cao, và các khán giả "tù nhân" là các hành khách phải ở lại trong một môi trường chờ được gọi lên máy bay với rất ít, hoặc không có gì khác ngoài các cửa hàng. Lịch bay cho phép nhà bán lẻ dự đoán được quốc tịch, độ tuổi, và những sở thích của các khách hàng tiềm năng tại các sân bay. Từ đó, điều chỉnh chuỗi sản phẩm bán phù hợp, và cho phép những hoạt động marketing nhắm đến thị trường mục tiêu tốt hơn (Newman và Lloyd, 1999). Tuy vậy, trong bán lẻ sân bay, khách hàng tiềm năng dường như khá giống nhau, họ thường có cảm giác lo lắng, bồn chồn, phấn khích, khiến cho họ phản ứng theo những cách không bình thường, và điều này có tác động cực kỳ quan trọng đến hành vi mua hàng của họ. Hiện tại, trong sân bay Tân Nhất, SASCO Shop là hệ thống cửa hàng bách hóa và lưu niệm - mỹ nghệ nằm ở cả ga quốc nội và ga quốc tế đáp ứng tối đa nhu cầu của hành khách với hơn 30.000 mặt hàng mang nét đặc trưng văn hóa của từng vùng miền Việt Nam.

\section{Phương pháp nghiên cứu}

\subsection{Cơ sở lý thuyết}

Các nghiên cứu gần đây về hành vi khách hàng (Dittmar và cộng sự, 1996; Tauber, 1972; Rook, 1987) đã chỉ ra rằng mặc dù khách hàng đã trở nên vô cùng phức tạp, đa số quyết định của họ lại theo hướng thôi thúc mua hàng một cách khó cưỡng lại được. Trong bối cảnh này, nhiều nhà nghiên cứu đã tìm ra rằng "thôi thúc mua (urge to shop)" như là một động lực để đi đây đi đó (Heung và $\mathrm{Qu}, 1998)$. "Có thể nghiên cúu, kiểm tra, cảm nhận và suy nghĩ về niềm vui có được tù việc mua một món hàng vốn là niềm vui với hàng triệu người, và đối với ho, đó chính là lý do, thậm chí là lý do chính để đi đó đây" (Lundberg, 1980). Do đó, khi đã diễn tả được quan hệ giữa du khách và mua sắm, các nhà làm chính sách cho các sân bay không còn coi việc hành khách di chuyển từ điểm này đến điểm khác là lý do duy nhất của sân bay dẫn đến việc các sân bay nổi lên như những trung tâm bán lẻ toàn cầu. Đa số các cửa hàng thương mại thường thấy ở các sân bay là các cửa hàng tiện lợi, đồ lưu niệm, cửa hàng miễn thuế, các dịch vụ ăn uống, quầy phục vụ hành khách, quầy giải trí, quảng cáo sân bay hay các dịch vụ viễn thông.

Có nhiều minh chứng cho thấy việc mua hàng ngẫu hứng tại sân bay chiếm tỷ trọng lớn, mà nếu các nhà bán lẻ hiểu rõ, và bám sát vào, sẽ có lợi cho việc tăng doanh số. Ai cũng biết đa số việc mua hàng sân bay là không dự định trước, Crawford và Melewar (1973) ước tính đến 70\% là mua ngẫu hứng. Các nhà nghiên cứu cũng chỉ ra rằng tác động mua ngẫu hứng rất khác nhau qua các phân khúc khách hàng, do bởi cả tác động tâm lý từ kinh nghiệm đi lại, lẫn sự hiện diện của các đặc điểm quy phạm (normative traits) khác nhau.

Mua ngẫu hứng là một đặc điểm chiếm nhiều sự chú ý của các nghiên cứu mua ngẫu hứng. Giả định căn bản của các nghiên cứu như vậy là con người rất khác nhau về khuynh hướng mua ngẫu hứng (Jones và cộng sự, 2003; Weun và cộng sự, 1998). 
Khuynh hướng mua ngẫu hứng (impulse buying tendency - IBT) là một trong những nhân tố quan trọng thúc đẩy khách hàng mua ngẫu hứng (Beatty và Ferrell, 1998). Người mua ngẫu hứng cao thường phản ứng nhanh với kích thích mua hàng (buying stimuli), và thường cởi mở hơn với các ý tưởng mua hàng không dự tính trước; vốn được kích hoạt ngay khi tiếp cận với một sản phẩm mong đợi; họ cũng bị hấp dẫn cảm xúc với sản phẩm cũng như sự hài lòng tức thời. Tuy vậy, ngay cả những khách hàng ngẫu hứng cao cũng không phải đáp ứng cho mọi kích thích mua hàng, vì nhiều nhân tố khác can thiệp vào như là tình trạng kinh tế, địa vị xã hội, hay áp lực thời gian can thiệp vào quá trình chuyển biến từ kỳ vọng ngẫu hứng (impulsive desire) đến hành vi ngẫu hứng (impulsive behavior) (Rook và Fisher, 1995). Điều này, do bởi thực tế là tại sân bay, hành khách thường có cảm giác lẫn lộn giữa căng thẳng, lo lắng và phấn khích, là điều họ thường không có. Trong môi trường sân bay, hành khách thường có hành vi xem lướt (browsing behavior), mặc dù điều này không chắc là dẫn đến mua ngẫu hứng.

Xem lướt trong cửa hàng chính là việc xem qua các mặt hàng bán lẻ nhằm có thông tin hay tiêu khiển mà không hề có ý định mua ngay (Bloch và cộng sự, 1986). Các nghiên cứu đã cho thấy xem lướt mà không có dự định gì có thể tạo ra một kinh nghiệm mua linh tinh rất thú vị (MacInnis và Price, 1987). Hơn nữa, điều này cũng ảnh hưởng khuynh hướng mua ngẫu hứng và có một tác động tích cực đến kích thích mua ngẫu hứng (Beatty và Ferrel, 1998). Nghiên cứu của Foroughi và cộng sự (2011) tìm ra quan hệ tích cực giữa khuynh hướng mua ngẫu hứng, niềm vui mua sắm, xem lướt trong cửa hàng và mua ngẫu hứng. Hành vi mua ngẫu hứng nhận được nhiều chú ý trong lý thuyết marketing và tâm lý trong khi có không nhiều nghiên cứu kiểm định tác động của xem lướt đến hành vi mua ngẫu hứng, đặc biệt trong môi trường sân bay. Mô hình nghiên cứu đề xuất (Hình 1) nhằm tìm hiểu quan hệ giữa khuynh hướng mua ngẫu hứng, xem lướt và mua ngẫu hứng.

Xem lướt cũng là một động lực xã hội cần phải tìm hiểu. Điều quan trọng là các nhà bán lẻ cần hiểu rõ quá trình suy nghĩ mà khách hàng trải qua trước khi họ quyết định mua một sản phẩm nào đó, bao gồm cả những điểm mà họ nhạy cảm với các thông điệp marketing.

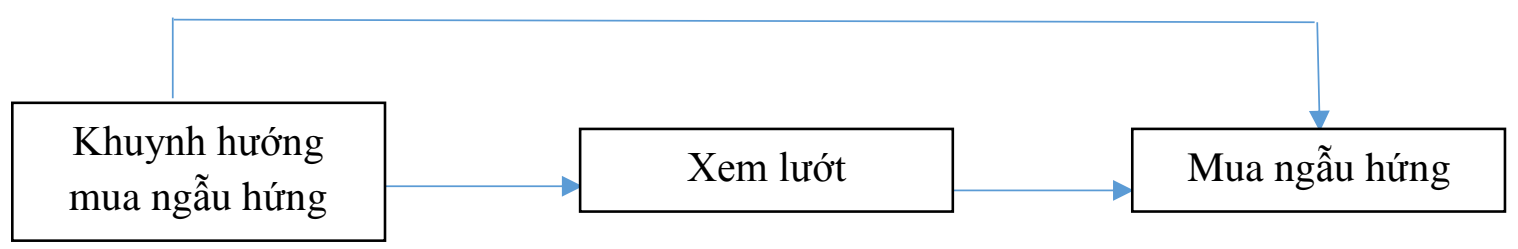

Hình 1. Mô hình khuynh hướng mua ngẫu hứng, xem lướt và mua ngẫu hứng

Nhiều nghiên cứu đã được thực hiện nhằm giải thích hành vi mua ngẫu hứng (Burroughs, 1996; Piron, 1993; Youn và Faber, 2000). Các nhà nghiên cứu marketing và bán lẻ đã phân loại hành vi mua ngẫu hứng như một tác nhân hiện thực và đầy quyền năng trong quy trình hành vi mua của khách hàng (Bayley và Nancarrow, 1998; Hausman, 2000). Mô hình quy trình ra quyết định mua của khách hàng (Engel và Blackwell, 1982) có 5 giai đoạn: nhận biết vấn đề/ nhu cầu, tìm kiếm 
các giải pháp khả thi, và đánh giá các giải pháp, mua và sử dụng sau mua và đánh giá lại việc chọn giải pháp. Coley (2002) cho rằng mô hình này thiếu giai đoạn mua ngẫu hứng, và đề xuất giai đoạn này xuất hiện ngay sau giai đoạn nhận diện vấn đề/ nhu cầu, sau đó đi thẳng đến các giai đoạn mua hàng, bỏ qua các giai đoạn tìm và đánh giá giải pháp. Các nhà nghiên cứu cũng đề xuất rằng nhận thức (cognitive) và tình cảm (affective) cũng cùng tác động cách thức và mức độ mà các cảm xúc hay các nguyên nhân dẫn đến tính ngẫu hứng.

Theo Jones và cộng sự (2003), mua ngẫu hứng là mua hàng ngay, mà không cân nhắc, đánh giá nhiều. Chien-Huang và Hung-Ming (2005) mô tả mua ngẫu hứng như hành vi mua hàng ngay, không dự định, ít cân nhắc, và không cưỡng lại được. Mua ngẫu hứng giống như khi khách hàng kích thích mua ngẫu hứng ngay rồi sau đó mới đánh giá việc mua có phù hợp không. Ẩn sau tính tự phát, mua ngẫu hứng là một kích thích mua cao trào, đầy kích động mà không cân nhắc đến hậu quả của quyết định mua.

Omar và Kent (2001) giải thích mua ngẫu hứng như một khái niệm nghiên cứu có ý nghĩa là khuynh hướng của người mua suy nghĩ và mua theo cách riêng của họ: hiện hữu, tự phát, không suy nghĩ, và ngay lập tức. Sharma và cộng sự (2010), Kacen và Lee (2002) khái niệm hành vi mua ngẫu hứng như là việc mua không có kế hoạch, có tính chất ra quyết định khá nhanh chóng, thiên lệch chủ quan để sở hữu ngay lập tức. Gutierrez (2004) mô tả mua ngẫu hứng là mua ngay khi khách hàng không thực sự tìm mua sản phẩm, và không hề dự định mua.

Một trong những nhân tố quan trọng tác động đến hành vi mua ngẫu hứng là môi trường, trong đó khách hàng đáp ứng với kích thích (Hà Nam Khánh Giao và Dương
Lê Phương Uyên, 2018). Tính đáp ứng này thay đổi theo đặc tính cá nhân, mỗi người có một khuynh hướng mua ngẫu hứng riêng, một số người có thiên hướng phản ứng cao với mua ngẫu hứng, trong khi số khác lại chẳng có phản ứng gì với các kích thích như vậy. Như vậy, khuynh hướng mua ngẫu hứng là mức độ mà một người thường hay mua hàng tự phát, không dự định và không chắc đáp ứng nhu cầu (Lin và Chuang, 2005). Khuynh hướng mua ngẫu hứng cũng được xem là biểu thị của tính ngẫu hứng (Rook và Fisher, 1995; Kacen và Lee, 2002; Weun và cộng sự, 1998; Youn và Faber, 2000; Cobb và Hoyer, 1986). Jones và cộng sự (2003) mở rộng khái niệm khuynh hướng mua ngẫu hứng từ chỗ tính chất cá nhân sang các biến liên quan sản phẩm hơn, cho thấy khuynh hướng mua ngẫu hứng một sản phẩm nhất định là kết quả của khuynh hướng mua ngẫu hứng chung và tính liên đới sản phẩm (product involvement).

Không như mô hình quy trình mua hàng dự định của Churchill và Peter (1998), quy trình mua ngẫu hứng bắt đầu bằng việc nhận thức sản phẩm (product awareness). Người mua ngẫu hứng bắt đầu xem lướt mà không có dự định gì về việc mua bất kỳ sản phẩm nào hay ghé vào bất kỳ một cửa hàng nào. Khi xem lướt, người mua bị kích thích, dẫn đến phát động việc mua ngẫu hứng. Kết quả là, người mua ra quyết định mua mà không tìm kiếm thông tin nào hay đánh giá các lựa chọn, chỉ cảm giác khó cưỡng lại việc mua (Giao và Mo, 2018).

Có thể thấy rằng xem lướt trong cửa hàng thường làm tăng khả năng mua ngẫu hứng, nghĩa là khách hàng càng xem lướt cửa hàng lâu hơn, thường họ kết thúc bằng việc mua ngẫu hứng (Beatty và Ferrell, 1998). Bầu không khí trong cửa hàng có vai 
trò quan trọng trong việc hấp dẫn khách hàng ở trong cửa hàng lâu hơn: trưng bày sản phẩm, các dấu hiệu, quầy kệ, trưng bày đặc biệt, khuyến mãi (Abratt và Goodey, 1990). Jarboe và McDaniel (1987) cho thấy người xem lướt thường mua hàng không dự định hơn những người không xem lướt. Omar và Kent (2001) nghiên cứu tại sân bay Gatwick cho thấy marketing và môi trường đều có tác động đến mua ngẫu hứng của khách hàng (35\% hành khách có xem lướt sẽ mua hàng).

\subsection{Mục tiêu nghiên cứu và giả thuyết nghiên cứu}

Các mục tiêu chính của nghiên cứu là (1) Kiểm tra quan hệ giữa khuynh hướng mua ngẫu hứng và mua ngẫu hứng, (2) Nghiên cứu tác động của khuynh hướng mua ngẫu hứng đến xem lướt trong cửa hàng, (3) Phân tích vai trò của xem lướt trong cửa hàng đối với mua ngẫu hứng, (4) Phân tích ảnh hưởng các yếu tố nhân chủng đến mua ngẫu hứng. Các giả thuyết bao gồm:

$\mathrm{H}_{1}$ : Có quan hệ tích cực giữa khuynh hướng mua ngẫu hứng và mua ngẫu hứng

$\mathrm{H}_{2}$ : Khuynh hướng mua ngẫu hứng càng cao thì xem lướt trong cửa hàng càng cao

$\mathrm{H}_{3}$ : Xem lướt trong cửa hàng càng cao thì mức mua ngẫu hứng càng cao

$\mathrm{H}_{4}$ : Có sự khác biệt giữa các yếu tố nhân chủng trong việc mua ngẫu hứng

\subsection{Phương pháp thu thập và phân tích dữ liệu}

Nghiên cứu chủ yếu dùng dữ liệu sơ cấp, được thu thập bằng bảng câu hỏi cấu trúc. Dữ liệu được thu thập từ 166 đáp viên có mua hàng ở sân bay. Bảng câu hỏi gồm các thành phần của 3 nhân tố mua ngẫu hứng, xem lướt và khuynh hướng mua ngẫu hứng. Bảng câu hỏi sử dụng thang đo Likert 5 khoảng, với 1 là hoàn toàn không đồng ý cho đến 5 là hoàn toàn đồng ý, cho 2 biến độc lập xem lướt và khuynh hướng mua ngẫu hứng, riêng biến phụ thuộc mua ngẫu hứng dùng thang đo 3 điểm gồm 1 (luôn luôn) đến 3 (chẳng bao giờ). Nghiên cứu định lượng sơ bộ thực hiện với 35 đáp viên. Khuynh hướng mua ngẫu hứng trích từ thang đo chuẩn của Rook và Fisher (1995), thang đo xem lướt trích từ nghiên cứu của Baron và Wass (1996). Phần mềm SPSS được sử dụng. Phân tích hồi quy để kiểm định trong đó biến độc là mua ngẫu hứng, $\mathrm{t}$ test và $A N O V A$ được sử dụng để phân tích sự khác biệt về tuổi, giới tính, thu nhập trong mua ngẫu hứng. Thống kê mô tả mẫu trong Bảng 1.

Bảng 1. Thống kê mô tả mẫu

\begin{tabular}{lcc}
\hline \multicolumn{1}{c}{ Đặc trưng } & Số lượng & Phần trăm (\%) \\
\hline Loại sản phẩm mua & & \\
Rượu, bia, thuốc lá & 42 & 25,3 \\
Sách báo & 68 & 41 \\
Đồ dùng vệ sinh & 5 & 3 \\
Quần áo & 19 & 11,4 \\
Đồ ăn uống & 127 & 76,5 \\
Đồ điện, điện tử & 27 & 16,3 \\
Linh kiện & 55 & 33,1 \\
Đồ linh tinh & 11 & 6,6
\end{tabular}




\section{Đặc trưng}

Số lượng

Phần trăm (\%)

\section{Giới tính}

Nam

88

53

Nữ

78

47

\section{Độ tuổi}

18-24

25-34

$35-45$

Trên 45

\section{Thu nhập}

Tới 10 triệu đồng/tháng

Trên 10 đến 25 triệu đồng/tháng

Trên 25 triệu đồng/tháng

\section{Loại hành khách}

Bay thường xuyên

Bay thỉnh thoảng

Kiểm định độ tin cậy bằng hệ số Cronbach's alpha. Cronbach's alpha của khuynh hướng mua ngẫu hứng là 0,788 và của xem lướt là 0,704 , đạt yêu cầu (Bảng 2) (Hà Nam Khánh Giao và Bùi Nhất Vương, 2019).

Bảng 2. Kiểm định độ tin cậy

\begin{tabular}{cc}
\hline Nhân tố & Cronbach's alpha \\
\hline Xem lướt & 0,704 \\
Khuynh hướng mua ngẫu hứng & 0,788 \\
\hline
\end{tabular}

\section{Kết quả và thảo luận}

\subsection{Kết quả}

Mục tiêu 1: Kiểm tra quan hệ giữa khuynh hướng mua ngẫu hứng và mua ngẫu hứng, Bảng 3 cho thấy khuynh hướng mua ngẫu hứng tác động trực tiếp đến mua ngẫu hứng của hành khách tại sân bay Tân Sơn Nhất, $\mathrm{R}^{2}$ hiệu chỉnh $=0,147$ cho thấy $14,7 \%$ mua ngẫu hứng là do tác động của khuynh hướng mua ngẫu hứng, $\beta=-0.390$ cho thấy quan hệ ngược chiều giữa 2 nhân tố.

Bảng 3. Hồi quy giữa IBT và mua ngẫu hứng

\begin{tabular}{lcccccccc}
\hline \multirow{2}{*}{ Mô hình } & \multicolumn{3}{c}{ Hệ số chưa chuẩn hóa $\begin{array}{c}\text { Hệ̀ số } \\
\text { chuẩn hóa }\end{array}$} & \multirow{2}{*}{$\mathrm{T}$} & \multicolumn{2}{c}{ Sig. } & \multicolumn{3}{c}{$\begin{array}{c}\text { Thống kê đa cộng } \\
\text { tuyến }\end{array}$} \\
\cline { 2 - 4 } & B & Sai số chuẩn & Beta & & & Độ chấp nhận & VIF \\
\hline 1 (Constant) & 2,669 & 0,198 & & 13,487 & 0,000 & 0,566 & 1,768 \\
Ibt & $-0,234$ & 0,043 & $-0,390$ & $-5,428$ & 0,000 & 0,394 & 2,536 \\
\hline
\end{tabular}


Biến phụ thuộc: mua ngẫu hứng

$\mathrm{R}^{2}$ hiệu chỉnh: 0,147

F: 29, 459

Sig: 0,000

Durbin - Watson: 2, 875

Mục tiêu 2: Nghiên cứu tác động của khuynh hướng mua ngẫu hứng đến xem lướt trong cửa hàng, Bảng 4 cho thấy IBT có tác động tích cực đến xem lướt $(\beta=$
$0,521 ; p=0,000), R^{2}=0,267$ cho thấy 27,2\% xem lướt do tác động của IBT. Như vậy, hành khách có IBT cao hơn sẽ xem lướt nhiều hơn tại các cửa hàng sân bay.

Bảng 4. Hồi quy giữa IBT và xem lướt

\begin{tabular}{|c|c|c|c|c|c|c|c|}
\hline \multirow[t]{2}{*}{ Mô hình } & \multicolumn{2}{|c|}{ Hệ số chưa chuẩn hóa } & \multirow{2}{*}{$\begin{array}{c}\begin{array}{c}\text { Hệ số } \\
\text { chuẩn hóa }\end{array} \\
\text { Beta }\end{array}$} & \multirow[t]{2}{*}{$\mathrm{T}$} & \multirow[t]{2}{*}{ Sig. } & \multicolumn{2}{|c|}{$\begin{array}{c}\text { Thống kê đa cộng } \\
\text { tuyến }\end{array}$} \\
\hline & B & Sai số chuẩn & & & & Độ chấp nhận & VIF \\
\hline 1 (Constant) & 2,380 & 0,278 & & 8,559 & 0,000 & 0,360 & 2,779 \\
\hline Ibt & 0,474 & 0,061 & 0,521 & 7,825 & 0,000 & 0,396 & 2,524 \\
\hline
\end{tabular}

Biến phụ thuộc: mua ngẫu hứng

$\mathrm{R}^{2}$ hiệu chỉnh: 0,267

F: 61,232

Sig: 0,000

Durbin - Watson: 3, 254

Mục tiêu 3: Phân tích vai trò của xem lướt trong cửa hàng đối với mua ngẫu hứng, Bảng 5 cho thấy xem lướt tác động nghịch chiều đến mua ngẫu hứng $(\beta=$ -
0,322, $\mathrm{p}=0,000), \mathrm{R}^{2}$ hiệu chỉnh = 0,099 cho thấy $9,9 \%$ mua ngẫu hứng là do tác động xem lướt trong cửa hàng, tác động này nhỏ.

Bảng 5. Hồi quy giữa xem lướt và mua ngẫu hứng

\begin{tabular}{|c|c|c|c|c|c|c|c|}
\hline \multirow[t]{2}{*}{ Mô hình } & \multicolumn{2}{|c|}{ Hệ số chưa chuẩn hóa } & \multirow{2}{*}{$\begin{array}{c}\begin{array}{c}\text { Hệ số } \\
\text { chuẩn hóa }\end{array} \\
\text { Beta }\end{array}$} & \multirow[t]{2}{*}{$\mathrm{T}$} & \multirow[t]{2}{*}{ Sig. } & \multicolumn{2}{|c|}{$\begin{array}{c}\text { Thống kê đa cộng } \\
\text { tuyến }\end{array}$} \\
\hline & $\mathrm{B}$ & Sai số chuẩn & & & & Độ chấp nhận & VIF \\
\hline 1 (Con & 2,579 & 0,224 & & 11,513 & 0,000 & 0,489 & 2,045 \\
\hline Ibt & $-0,213$ & 0,049 & $-0,322$ & $-4,363$ & 0,000 & 0,527 & 1,898 \\
\hline
\end{tabular}

Biến phụ thuộc: mua ngẫu hứng

$\mathrm{R}^{2}$ hiệu chỉnh: 0,099

F: 19,035

Sig: 0,000

Durbin - Watson: 1, 982 
Mục tiêu 4: Phân tích ảnh hưởng các yếu tố nhân chủng đến mua ngẫu hứng, $\mathrm{t}$ - test được dùng để kiểm định tác động của giới tính đến mua ngẫu hứng. Bảng 6 cho thấy $\mathrm{p}$ value $=0,918>0,05$, không có sự khác biệt trong mua ngẫu hứng giữa nam và nữ.

Bảng 6. Kiểm định sự khác biệt theo giới tính

\begin{tabular}{|c|c|c|c|c|c|c|c|c|c|c|}
\hline & \multicolumn{5}{|c|}{ Kiểm định Levene's } & \multicolumn{5}{|c|}{ t-test giá trị trung bình } \\
\hline & \multirow{2}{*}{$\mathrm{F}$} & \multirow{2}{*}{\multicolumn{2}{|c|}{ Sig. }} & \multirow{2}{*}{$\mathrm{T}$} & \multirow{2}{*}{ Df } & \multirow{2}{*}{$\begin{array}{l}\text { Sig. (2 } \\
\text { chiều) }\end{array}$} & \multirow{2}{*}{$\begin{array}{l}\text { Khác } \\
\text { biệt } \\
\text { trung } \\
\text { bình }\end{array}$} & \multirow{2}{*}{$\begin{array}{l}\text { Sai biệt } \\
\text { độ lẹ̣ch } \\
\text { chuẩn }\end{array}$} & \multicolumn{2}{|c|}{$\begin{array}{c}\text { Độ tin cậy } \\
95 \%\end{array}$} \\
\hline & & & & & & & & & $\begin{array}{l}\text { Thấp } \\
\text { hơn }\end{array}$ & $\begin{array}{l}\text { Cao } \\
\text { hơn }\end{array}$ \\
\hline \multirow{2}{*}{$\begin{array}{l}\text { Mua } \\
\text { ngâ̂u } \\
\text { hứng }\end{array}$} & $\begin{array}{l}\text { Giả định } \\
\text { phương sai } \\
\text { bằng nhau }\end{array}$ & 1,273 & 0,261 & 0,103 & 164 & 0,918 & 0,010 & 0,093 & $-0,175$ & 0,194 \\
\hline & $\begin{array}{l}\text { Giả định } \\
\text { phương sai } \\
\text { không bằng } \\
\text { nhau }\end{array}$ & & & 0,102 & 156,904 & 0,919 & 0,010 & 0,094 & $-0,176$ & 0,195 \\
\hline
\end{tabular}

ANOVA một chiều được sử dụng để phân tích tác động của nhóm tuổi và thu nhập đến mua ngẫu hứng (Bảng 7 và Bảng 8). Kết quả lần lược $\mathrm{p}$ value $=0,326>0,05$ và $p$ value $=0,657>0,05$, nghĩa là không có sự khác biệt giữa các nhóm tuổi và các nhóm thu nhập trong mua ngẫu hứng tại sân bay.

Bảng 7. Kiểm định sự khác biệt theo nhóm tuổi

\begin{tabular}{lccccc}
\hline \multicolumn{5}{c}{ Mua ngẫu hứng } \\
\hline & $\begin{array}{c}\text { Tổng bình } \\
\text { phương }\end{array}$ & Df & $\begin{array}{c}\text { Trung bình } \\
\text { bình phương }\end{array}$ & F & Sig. \\
\hline Giữa các nhóm & 1,246 & 3 & 0,415 & 1,163 & 0,326 \\
Trong các nhóm & 57,845 & 162 & 0,357 & & \\
Tổng & 59,090 & 165 & & & \\
\hline
\end{tabular}

Bảng 8. Kiểm định sự khác biệt theo thu nhập

\begin{tabular}{lccccc}
\hline \multicolumn{5}{c}{ Mua ngẫu hứng } \\
\hline & $\begin{array}{c}\text { Tổng bình } \\
\text { phương }\end{array}$ & Df & $\begin{array}{c}\text { Trung bình } \\
\text { bình phương }\end{array}$ & F & Sig. \\
\hline Giữa các nhóm & 0,303 & 2 & 0,152 & 0,420 & 0,657 \\
Trong các nhóm & 58,787 & 163 & 0,361 & & \\
Tổng & 59,090 & 165 & & & \\
\hline
\end{tabular}




\section{Kiểm định giả thuyết}

Các giả thuyết nghiên cứu được kiểm định như trong Bảng 9 .

Bảng 9. Các giả thuyết nghiên cứu và kiểm định

\begin{tabular}{lc}
\hline \multicolumn{1}{c}{ Giả thuyết } & Kiểm định \\
\hline $\mathrm{H}_{1}$ : Có quan hệ tích cực giữa khuynh hướng mua ngẫu hứng và mua ngẫu & Bác bỏ \\
hứng & \\
$\mathrm{H}_{2}$ : Khuynh hướng mua ngẫu hứng càng cao thì xem lướt trong cửa hàng & Chấp nhận \\
càng cao & \\
$\mathrm{H}_{3}$ : Xem lướt trong cửa hàng càng cao thì mức mua ngẫu hứng càng cao & Chấp nhận \\
$\mathrm{H}_{4}$ : Có sự khác biệt giữa các yếu tố nhân chủng trong việc mua ngẫu hứng & Bác bó \\
\hline
\end{tabular}

\subsection{Thảo luận}

Kết quả nghiên cứu cho thấy khuynh hướng mua ngẫu hứng tác động ngược chiều đến mua ngẫu hứng, như vậy, người mua ngẫu hứng không hẳn đã mua ngẫu hứng tại sân bay. Phát hiện lý thú này có thể do, tại sân bay, hành khách chịu tác động bởi hai khuynh hướng cảm xúc đến hành vi mua hàng của họ (Thomas, 1997): một là sự tăng cao mức căng thẳng do họ rời khỏi những việc hàng ngày của mình, hai là tăng mức mức phấn khích. Ngoài ra, phát hiện lý thú này có thể do những tác động đa chiều của đại dịch Covid-19, làm cho các hành vi tiêu dùng thay đổi, mà chưa được cập nhật trong lý thuyết cũng như kiểm định trong thực tế; theo đó, người có khuynh hướng mua ngẫu hứng càng cao, càng phải chuyển hướng sang các phương thức thương mại khác, thay vì mua ngẫu hứng tại cửa hàng, mà đặc biệt là trong môi trường sân bay.

Người có khuynh hướng mua ngẫu hứng càng cao thì xem lướt trong cửa hàng càng cao. Xem lướt là hành động thường thấy tại các sân bay, vì nhiều lý do, mà lý do hiển nhiên nhất là hành khách thường chịu áp lực thời gian, do vậy, xem lướt chính là cách để tránh bớt áp lực đó. Thời gian sã̃n có chính là một tác nhân tình huống
(Belk, 1975). Trong khoảng thời gian có sẵn, hành khách thường chọn xem lướt hơn là ngồi chờ một cách buồn chán (Hirshman và Holbrook, 1982). Xem lướt không hẳn dẫn đến mua hàng, do đó, kích thích hoạt động xem lướt là phát hiện chính của nghiên cứu này. Ảnh hưởng của kích thích bên ngoài và tình trạng bên trong (hành khách) là điểm nổi bật ở các sân bay (Omar, 2002). Các cuộc phỏng vấn ngắn với hành khách tại sân bay cho thấy họ thường kích động cao, lo lắng nhiều về chuyến bay sắp diễn ra (suy nghĩ về việc xa nhà, các thủ tục bay, coi chừng hành lý, cẩn thận vé và các giấy tờ cần thiết như chứng minh nhân dân hay hộ chiếu, tìm đúng cổng bay, và lên máy bay đúng giờ, ...), những việc này tác động nhiều đến hành vi mua hàng. Ngoài ra, các yếu tố nhân chủng không ảnh hưởng gì đến hành vi mua ngẫu hứng.

Mua ngẫu hứng diễn ra trong cửa hàng, nhưng nhiều minh chứng cho thấy đó không phải hình thức chính của hành vi mua hàng. Do vậy, các nhà bán lẻ cần chú trọng thúc đẩy giây phút kích thích mua hàng (spur of moment purchases), cần tăng cường giới thiệu những gì không mong đợi (Newby, 1993) giữa những thương hiệu nổi tiếng, cần tăng cường không khí lễ hội bằng cách thiết kế bầu không khí dịch vụ hấp dẫn, tăng 
cường các yếu tố bất ngờ, cuốn hút (Peter và Olson, 1994), cần thiết kế cách dẫn dắt sáng tạo giữa các món hàng, cần trưng bày hấp dẫn cho nhu cầu mua cảm giác (hedonic needs). Muốn vậy, nhà bán lẻ cần kích thích mua ngẫu hứng dựa trên việc phân khúc khách hàng, có thể do chính họ nghiên cứu, hay dựa vào những tổ chức nghiên cứu chuyên nghiệp. Các nhà quản lý sân bay Tân Sơn Nhất có thể nghiên cứu thêm cách làm của đồng nghiệp tại sân bay Incheon (Hàn Quốc) trong việc tổ chức những động tác marketing bằng những đoàn diễn viên trong trang phục truyền thống đi vòng khắp các nhà ga, và tập trung trình diễn một vài tiết mục giới thiệu truyền thống tại những nơi mua bán sầm uất nhất các nhà ga, vào những khung giờ nhất định, thu hút đông đảo người xem; cũng có thể nghiên cứu cách làm của sân bay Chang-gi (Singapore) trong việc tạo ra môi trường thiên nhiên trong các nhà ga.

Nghiên cứu cũng cho thấy chỉ riêng xem lướt không dẫn đến mua ngẫu hứng, mà là một phức hợp các yếu tố môi trường và tâm lý bên trong hành khách. Do vậy, các nhà bán lẻ sân bay nên nhận biết tình trạng tâm lý của nhiều loại hành khách khác nhau và cố gắng tạo ra môi trường có thể làm giảm căng thẳng của hành khách, hay ít nhất, duy trì độ phấn kích bình thường, trong khi vẫn tạo động lực mua ngẫu hứng bằng việc giảm hay loại bỏ các trở lực mua hàng. Nghiên cứu này được thực hiện trong những tháng đầu năm 2021 tại sân bay Tân Sơn Nhất, khi mà nỗi lo lắng về tình trạng bùng phát dịch bệnh Covid-19 vẫn còn, nhất là sau khoảng thời gian giữa tháng $1 / 2021$ đến cuối tháng 2/2021, sân bay Tân Sơn Nhất được cảnh báo nguy cơ lây nhiễm do sự cố dương tính của một vài nhân viên, rồi đến giữa cuối tháng 4/2021, khi lượng hành khách tăng đột biến do các chương trình kích cầu du lịch, hành khách mệt mỏi, lo lắng vì những thủ tục phòng chống dịch bệnh như khai báo y tế, đảm bảo khoảng cách, lại còn thêm việc dồn ứ khách tại các quầy thủ tục, kiểm tra an ninh, soi chiếu tại sân bay Tân Sơn Nhất (Hà Mai, 2021), xem lướt không còn là xem lướt như trước đây, mà chỉ là vội vã lướt qua các quầy hàng trên đường tìm đến các cổng ra máy bay cho kịp giờ. Tất nhiên, xem lướt như vậy sẽ không dẫn đến hành vi mua ngẫu hứng tích cực như kết quả của các nghiên cứu trước có liên quan.

Newmann và Jones (1999) tìm ra tỷ lệ hành khách viếng thăm cửa hàng tại sân bay là khoảng $50 \%$, trong đó xem lướt không dự định là $15 \%$, và chỉ $35 \%$ trong số này mua ngẫu hứng. Do vậy, các nhà bán lẻ sân bay cần trang bị các chiến lược nhằm làm tăng mức độ xem lướt, và dự định mua hàng. Trong bối cảnh của sân bay Tân Sơn Nhất, nhà bán lẻ SASCO chỉ có thể làm tất cả những gì có thể, bên cạnh đó, cũng rất cần sự hỗ trợ của các đơn vị khác có liên quan trong sân bay như Tổng công ty Cảng hàng không Việt Nam (ACV) xây dựng kế hoạch và triển khai tăng cường công tác bảo đảm an ninh, an toàn hàng không, chất lượng dịch vụ và phòng chống dịch Covid-19, sắp xếp thêm lực lượng thanh niên tình nguyện đã được huấn luyện để hỗ trợ khách hàng từ cửa $\mathrm{ra} /$ vào cho tới khi khách vào quầy check-in. Bên cạnh đó, $\mathrm{ACV}$ cần làm việc với điều hành bay, phối hợp với các cơ quan, đơn vị rà soát công tác điều phối slot (lượt cất hạ cánh), điều chỉnh số lượng slot, phân bổ dàn đều slot trong tất cả khung giờ để đảm bảo các hãng không để trùng giờ cất cánh, dồn nhiều chuyến bay vào cùng thời điểm gây tình trạng tắc nghẽn cục bộ (Hà Mai, 2021). 


\section{Kết luận}

Bán lẻ sân bay đang ngày càng phát triển. Hành vi mua ngẫu hứng tại sân bay vẫn chưa được chú ý nhiều trong lý thuyết, đặc biệt tại sân bay Tân Sơn Nhất. Mô hình nghiên cứu đề xuất bao gồm 03 thành phần chính: mua ngẫu hứng, xem lướt và khuynh hướng mua ngẫu hứng, được kiểm định liên quan trong môi trường sân bay. Kết quả phân tích cho thấy khuynh hướng mua ngẫu hứng cao hơn chưa hẳn dẫn đến mua ngẫu hứng cao hơn, nhưng dẫn đến xem lướt trong cửa hàng cao hơn tại sân bay Tân Sơn Nhất. Ngoài ra, kết quả cũng cho thấy mức độ mua ngẫu hứng cũng không tăng cùng xem lướt tại sân bay Tân Sơn Nhất. Kiểm định khác biệt không cho thấy các biến nhân chủng có ảnh hưởng đến mua ngẫu hứng tại sân bay Tân Sơn Nhất. Phân tích dữ liệu cho thấy môi trường là nhân tố quan trọng dẫn đến mua ngẫu hứng. Từ đó, nghiên cứu cũng đề xuất một số hàm ý quản trị đến các nhà bán lẻ tại sân bay Tân Sơn Nhất, nhằm nâng cao mua ngẫu hứng. Theo đó, cần tăng cường xem lướt theo cách có thể khuyến khích mua thật sự tại sân bay, và không cần lưu ý nhiều đến tuổi, giới tính, thu nhập của hành khách mua ngẫu hứng.

Mặc dù đã hoàn thành các mục tiêu đề ra, nghiên cứu vẫn có những hạn chế nhất định: (1) Thu thập dữ liệu bằng bảng hỏi tại sân bay Tân Sơn Nhất chưa hẳn là phương pháp tốt, khi hành khách đều có chung tâm trạng bồn chồn, không chú tâm, sẽ có những câu trả lời qua loa, thiên lệch, (2) Phương pháp chọn mẫu thuận tiện, làm cho độ tin cậy và khái quát hóa chưa cao, (3) Một số nhân tố khác tác động đến mua ngẫu hứng: môi trường, văn hóa, tâm trạng, cảm xúc, ... đặc biệt trong môi trường sân bay, chưa được đề cập trong nghiên cứu này, (4) Các lý thuyết nền cùng các nghiên cứu đối sánh được đề cập trong nghiên cứu đều có thời gian đăng tải trước đại dịch Covid-19, trong khi nghiên cứu được thực hiện trong những tháng đầu 2021, có thể nhiều hành vi tiêu dùng, đặc biệt là mua ngẫu hứng đã có những thay đổi, và chưa được cập nhật vào lý thuyết, cũng như chưa được kiểm định thực tế, (5) Phương pháp hồi quy tuần tự để giải quyết từng mục tiêu riêng lẻ chưa cho thấy bối cảnh tác động chung cùng lúc của các nhân tố, cần sử dụng phương pháp mô hình cấu trúc tuyến tính (Structural Equation Modelling - SEM) để giải quyết tốt hơn. Đó cũng chính là những đề xuất cho các nghiên cứu trong tương lai.

\section{Tài liệu tham khảo}

Abratt, R., and Goodey, S. D. (1990). Unplanned Buying and In-Store Stimuli in Supermarkets. Managerial and Decision Economics, 11(2): 111-121.

Baron, S., and Wass, K. (1996). Towards an understanding of airport shopping behavior. The International Review of Retail, Distribution and Consumer Research, 6(3): 301-322. DOI: $10.1080 / 09593969600000026$

Bayley, G. D. and Nancarrow, C. (1998). Impulse purchasing: a qualitative exploration of the phenomenon. Qualitative Market Research: An International Journal, 1, 99-114.

DOI:10.1108/13522759810214271

Beatty, S. E., and Ferrell, M. E. (1998). Impulse buying: Modeling its precursors. Journal of Retailing, 74(2): 169-191.

https://doi.org/10.1016/S00224359(99)80092-X

Belk, R. W. (1975). Situational Variables and Consumer Behavior. Journal of 
Consumer Research, 2(3): 157 - 164.

https://doi.org/10.1086/208627

Bloch, P. H., Sherrell, D. L., and Ridgway,

N. M. (1986). Consumer search: An extended framework. Journal of Consumer Research, 13(1): 119-126. https://doi.org/10.1086/209052

Burroughs, J. E. (1996). Product Symbolism, Self Meaning, and Holistic Matching: the Role of Information Processing in Impulsive Buying. Advances in Consumer Research, 23, 463-469.

Chien-Huang, L., and Hung-Ming, L. (2005). An exploration of Taiwanese adolescents' impulsive buying tendency. Adolescence, 40(157): 215223.

Churchill, G. A., and Peter, J. P. (1998). Marketing: Creating Value for Customers (2 $2^{\text {nd }}$ Ed.). New York: McGraw-Hill/Irwin.

Cobb, C. J., and Hoyer, W. D. (1986). Planned versus impulse purchase behavior. Journal of Retailing, 62(4): 384-409.

Coley, A. L. (2002). Affective and cognitive processes involved in impulse buying (Unpublished Master's thesis). The University of Georgia, Athens.

Crawford, G., and Melewar, T. C. (2003). The importance of impulse purchasing behaviour in the international airport environment. Journal of Consumer Behaviour, $3(1)$ : 85-98.

DOI:10.1002/cb.124

Dittmar, H., Beattie, J., and Friese, S. (1996). Objects, decision considerations and self- images in men's and women's impulse purchases. Acta Psychologica, 93(1-3): 187-206. https://doi.org/10.1016/0001-

\section{8(96)00019-4}

Đỗ Nga (2020). Việt Nam là một trong những thị trường bán lẻ hấp dẫn nhất toàn cầu. https://congthuong.vn/vietnam-la-mot-trong-nhung-thi-truongban-le-hap-dan-nhat-toan-cau140704.html, truy cập ngày 22/12/2020.

Engel, J. F., and Blackwell, R. D. (1982). Consumer behavior (4 ${ }^{\text {th }}$ Ed.). Chicago: Dryden Press.

Foroughi, A., Buang, A., and Sherilou, M. (2011). Exploring impulse buying behavior among Iranian tourists in Malaysia. Journal of Global Business and Economics, 3(1): 187-195.

Giao, H. N. K., and Mo, N. T. H. (2018). Factors influencing consumers' impulse television buying decision at Best Buy Vietnam (BBVN). Global and Stochastic Analysis, 5(6): 353-369. DOI: 10.31219/osf.io/cgz2x.

Gutierrez, B. P. (2004). Determinants of planned and impulse buying: The case of the Philippines. Asia-Pacific Management Review, 9(6): 1061-1078. DOI:10.6126/APMR.2004.9.6.03

Hà Mai (2021). Dồn lực tháo ùn nghẽn ở sân bay Tân Sơn Nhất dịp lễ 30/4 - 1/5. https://thanhnien.vn/tai-chinh-kinhdoanh/don-luc-thao-un-nghen-o-sanbay-tan-son-nhat-dip-le-304-15-

1373028.html, truy cập ngày 25/04/2021.

Hà Nam Khánh Giao và Bùi Nhất Vương (2019). Giáo trình Cao hoc Phuoong pháp Nghiên cúu Khoa hoc trong Kinh doanh - Cập nhật SmartPLS. Hà Nội: $\mathrm{Nxb}$ Tài chính.

Hà Nam Khánh Giao và Dương Lê Phương Uyên (2018). Tác động của bầu không khí trong siêu thị đến hành vi của khách 
hàng: trường hợp siêu thị Coopmart. Tạp chí Quản lý Kinh tế, 88(5,6): 1726. DOI: $10.31219 /$ osf.io/sfx76.

Hausman, A. (2000). A multi-method investigation of consumer motivation in impulse buying behavior. Journal of Consumer Marketing, 17(5): 403-426. DOI: $10.1108 / 07363760010341045$

Heung, V. C. S., and Qu, H. (1998). Tourism shopping and its contributions to Hong Kong. Tourism Management, 19(4): 383-386. DOI: 10.1016/s02615177(98)00029-6

Hirschman, E. C., and Holbrook, M. B. (1982). Hedonic consumption: Emerging concepts, Methods and propositions. Journal of Marketing, 46(3): 92-101. https://doi.org/10.2307/1251707

Jarboe, G. R., and Mc Daniel, C. D. (1987). A profile of browsers in regional shopping malls. Journal of the Academy of Marketing Science, 15, 4653. DOI:10.1007/BF02721953

Jones, M. A., Reynolds, K. E., Weun, S., and Beatty, S. E. (2003). The productspecific nature of impulse buying tendency. Journal of Business Research, 56(7): 505-511. https://doi.org/10.1016/S01482963(01)00250-8

Kacen, J. J., and Lee, J. A. (2002). The influence of culture on consumer impulsive buying behavior. Journal of Consumer Psychology, 12(2): 163-176. https://doi.org/10.1207/S15327663JC P1202_08

Lin, C., and Chuang, S. (2005). The effect of individual differences on adolescents' impulsive buying behavior. Adolescence, 40(159): 551558.
Lundberg, D. E. (1980). The tourist business. Boston, CBI Publishing Company.

MacInnis, D. J., and Price, L. L. (1987). The role of imagery in information processing: Review and extensions. The Journal of Consumer Research, 13(4): 473-491.

Newby, P. (1993). Shopping as leisure. In Retail change: Contemporary issues (Bromley, R.D., Thomas, C.J (Eds.)). London: UCL Press, 208-227.

Newman, S. J., and Lloyd-Jones, T. (1999). Airport and travel terminal retailing: Strategies, Trends and market dynamics. London: Ravenfox publishing.

Omar, O. (2002). Airport Retailing: Examining Airline Passengers' Impulsive Shopping. Journal of Euromarketing, 11(1): 87-105. DOI: https://doi.org/10.1300/J037v11n01_0 5.

Omar, O., and Kent, A. (2001). International airport influences on impulsive shopping: Trait and normative approach. International Journal of Retail và Distribution Management, 29(5): 226-235. DOI:10.1108/09590550110390887

Peter, J. P., and Olson, J. C. (1994). Understanding consumer behavior. Burr Ridge, IL: Irwin.

Piron, F. (1993). A comparison of emotional reactions experienced by planned, unplanned and impulse purchasers. Advances in Consumer Research, 20, 341-344.

Rook, D. W., and Fisher, R. J. (1995). Normative Influences on Impulsive Buying Behavior. The Journal of Consumer Research, 22(3): 305-313. 
Rook, D. W. (1987). The Buying Impulse. Journal of Consumer Research, 14(2): 189-199. DOI: https://doi.org/10.1086/209105

Sharma, P., Sivakumaran, B., and Marshall, R. (2010). Impulse buying and variety seeking: A trait-correlates perspective. Journal of Business Research, 63(3): 276-283.

DOI: https://doi.org/10.1016/j.jbusres.2009. 03.013

Tauber, E. M. (1972). Why do people shop? Journal of Marketing, 36(4): 46-49. DOI: https://doi.org/10.2307/1250426

Thomas, D. (1997). Retail and leisure developments at London Gatwick,
Commercial airport. London: British Airport Authority, 38 - 41.

Walsh, S., and McColl, C. (1994). Opportunities blossom for airport shopping centres. Airport technology international, Annual review, 63-65.

Weun, S., Jones, M. A., and Beatty, S. E. (1998). Development and validation of the impulse buying tendency scale. Psychological Reports, 82(3): 11231133.

Youn, S., and Faber, R. J. (2000). Impulsive buying: Its relation to personality traits and cues. Advances in Consumer Research, 27, 179-185. 